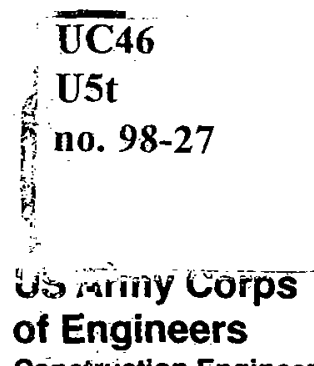

Construction Engineering

Research Laboratories
US-CE-C Properly :

United States Governm

\title{
Reflector Microphones for Field Recording of Natural Sounds
}

by

George W. Swenson, Jr.

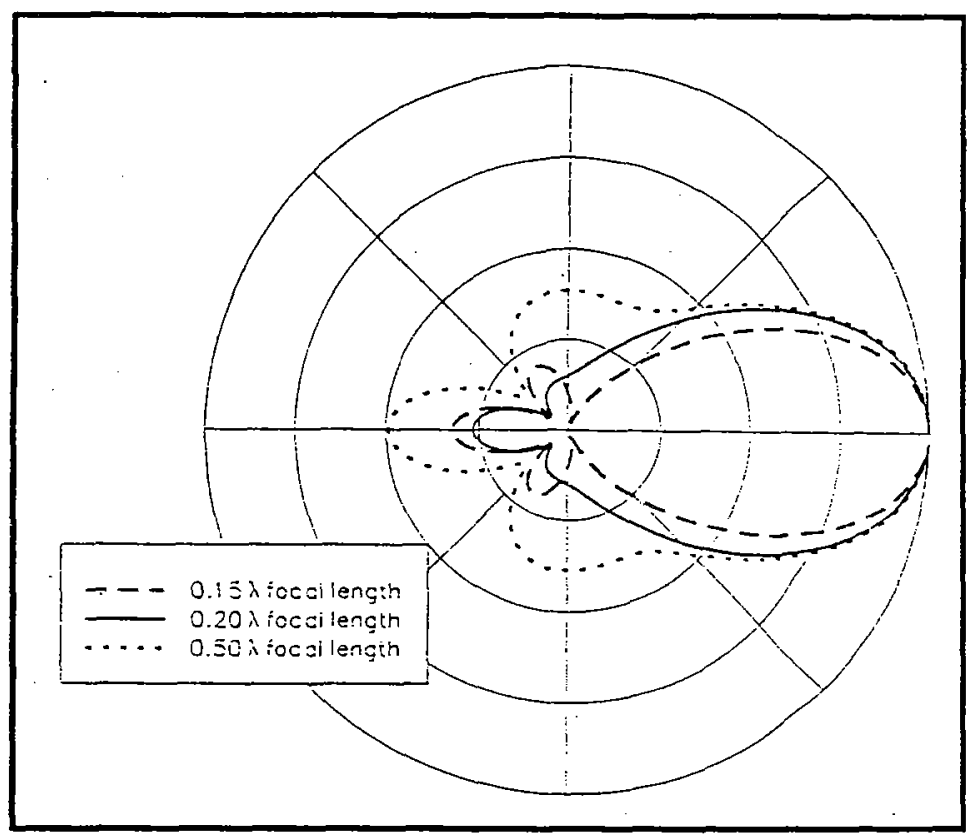

Directional microphone systems for field recording of sounds in the air usually involve either a parabolic reflector to focus the sound waves on the microphone (transducer) element, or a linear array of transducers so phased as to respond preferentially to sounds from one directional sector. The latter system (the "shotgun" microphone) can be analyzed in a fairly straightforward manner. The reflector system involves a structure comparable to a wavelength in linear dimension, and is therefore not susceptible to the conventional approximate methods of computation.

Recently developed computational techniques now permit exact calculation of the directional responses of small reflectors. This work computed the characteristics of a number of small microphone reflectors and linear microphone arrays. A flat reflector microphone for the' low-frequency range of 10 to $40 \mathrm{~Hz}$ was also described. The study concluded that no directional microphone can, in practice, reproduce sounds with fidelity to the sounds as emitted by the source. 
The contents of this report are not to be used for advertising, publication, or promotional purposes. Citation of trade names does not constitute an official endorsement or approval of the use of such commercial products. The findings of this report are not to be construed as an official Department of the Army position, unless so designated by other authorized documents.

DESTROY THIS REPORT WHEN IT IS NO LONGER NEEDED

DO NOT RETURN IT TO THE ORIGINATOR 
Public reporting burden for this collection of information is estimated to average 1 hour per response, including the time for reviewing instructions, searching existing data sources, gathering and maintaining the data needed, and completing and reviewing the collection of information. Send comments regarding this burden estimate or any other aspect of this collection of information, including suggestions for reducing this burden, to Washington Headquarters Services, Directorate for information Operations and Reports, 1215 Jeflerson Davis Highway, Suite 1204, Arlington, VA 22202-4302, and to the Office of Management and Budget, Paperwork Reduction Project (0704-0188), Washington, DC 20503.

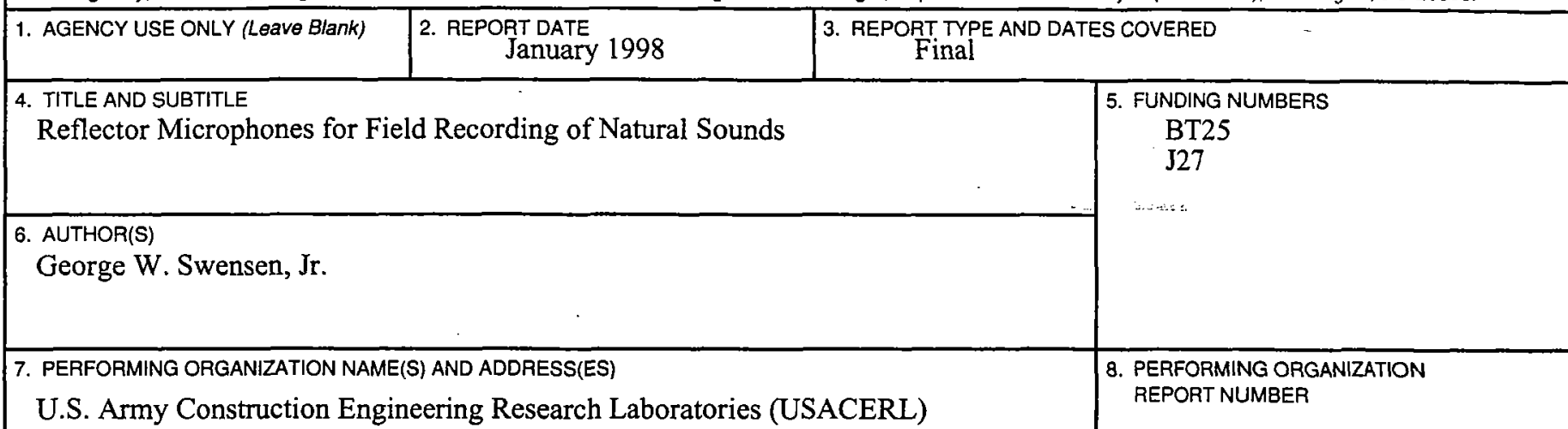

P.O. Box 9005

Champaign, IL 61826-9005

TR 98/27

9. SPONSORING / MONITORING AGENCY NAME(S) AND ADDRESS(ES)

U.S. Army Center for Health Promotion and Preventive Medicine (USACHPPM)

ATTN: MCHB-DC-EN

5158 Blackhawk Road

Aberdeen Proving Ground, MD 21010-5422.

11. SUPPLEMENTARY NOTES

Copies are available from the National Technical Information Service, 5285 Port Royal Road, Springfield, VA 22161.

12a. DISTRIBUTION/AVAILABILITY STATEMENT

12b. DISTRIBUTION CODE

Approved for public release; distribution is unlimited.

13. ABSTRACT (Maximum 200 words)

Directional microphone systems for field recording of sounds in the air usually involve either a parabolic reflector to focus the sound waves on the microphone (transducer) element, or a linear array of transducers so phased as to respond preferentially to sounds from one directional sector. The latter system (the "shotgun" microphone) can be analyzed in a fairly straightforward manner. The reflector system involves a structure comparable to a wavelength in linear dimension, and is therefore not susceptible to the conventional approximate methods of computation. Recently developed computational techniques now permit exact calculation of the directional responses of small reflectors. This work computed the characteristics of a number of small microphone reflectors and linear microphone arrays. A flat reflector microphone for the low-frequency range of 10 to $40 \mathrm{~Hz}$ was also described. The study concluded that no directional microphone can, in practice, reproduce sounds with fidelity to the sounds as emitted by the source.

\begin{tabular}{|c|c|c|c|}
\hline \multirow{2}{*}{\multicolumn{3}{|c|}{$\begin{array}{l}\text { 14. SUBJECT TERMS } \\
\text { Reflectors } \\
\text { Microphones } \\
\text { Sound recording }\end{array}$}} & \multirow{2}{*}{$\begin{array}{l}\text { 15. NUMBER OF PAGES } \\
18 \\
\text { 16. PRICE CODE }\end{array}$} \\
\hline & & & \\
\hline $\begin{array}{l}\text { 17. SECURITY CLASSIFICATION } \\
\text { OF REPORT } \\
\text { Unclassified }\end{array}$ & $\begin{array}{l}\text { 18. SECURITY CLASSIFICATION } \\
\text { OF THIS PAGE } \\
\text { Unclassified }\end{array}$ & $\begin{array}{l}\text { 19. SECURITY CLASSIFICATION } \\
\text { OF ABSTRACT } \\
\text { Unclassified }\end{array}$ & $\begin{array}{l}\text { 20. LIMITATION OF } \\
\text { ABSTRACT }\end{array}$ \\
\hline NSN 7540-01-280-5500 & & & $\begin{array}{l}\text { Form } 298 \text { (Rev. 2-89) } \\
\text { by ANSI Std 239-18 }\end{array}$ \\
\hline
\end{tabular}




\section{Foreword}

This study was conducted for U.S. Army Center for Health Promotion and Preventive Medicine (USACHPPM) under Project 4A161102BT25, "Environmental Research-Corps of Engineers"; Work Unit J27, "Remote Activity Monitoring.” The technical monitor was William Russell, MCHB-DCEEN.

The work was performed by the Planning and Mission Impact Division (LL-P) of the Land Management Laboratory (LL), U.S. Army Construction Engineering Research Laboratories (USACERL). The USACERL principal investigator was Dr. Larry L. Pater. Dr. George W. Swenson, Jr. is associated with the Department of Electrical and Computer Engineering, University of Illinois, Urbana. The computations presented here were performed using code written by Soon Young Lee and Alona Boag. Some of the graphs were prepared by Jonathan Benson. Dr. Harold E. Balbach is Chief, CECER-LL-P; Dr. William D. Severinghaus is Operations Chief, CECER-LL; and William D. Goran is the responsible Technical Director, CECER-LL. The USACERL technical editor was William J. Wolfe, Technical Resources.

COL James A. Walter is Commander and Dr. Michael J. O'Connor is Director of USACERL. 


\section{Contents}

SF 298 ................................................................................................................................. 1

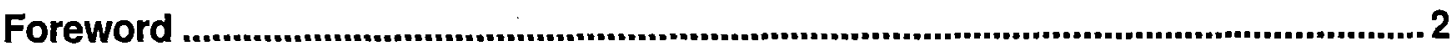

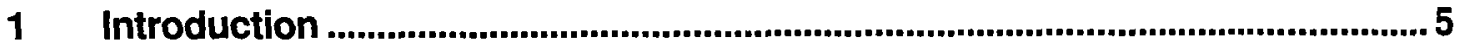

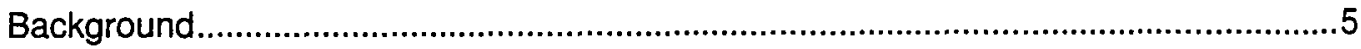

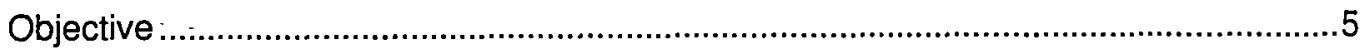

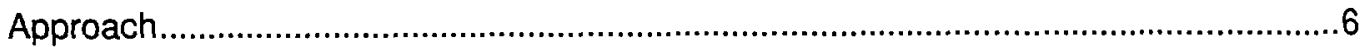

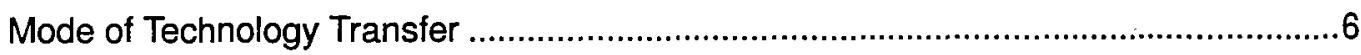

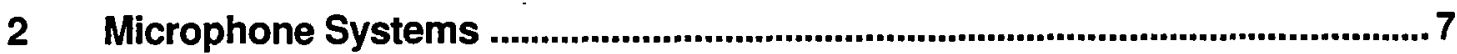

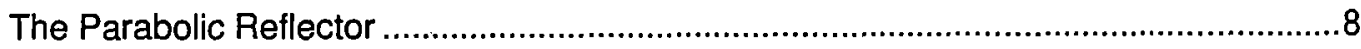

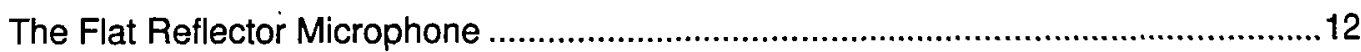

End-Fire Microphones ................................................................................. 14

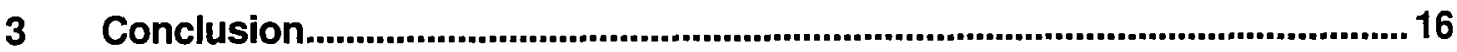

References ................................................................................................................... 17

Distribution 


\section{Introduction}

\section{Background}

For observing or recording of sound from a discrete direction, particularly in the outdoor environment, it is appropriate to use a microphone system that receives preferentially from that direction, to reduce the influence of extraneous noise from other directions and to realize a gain in the power received from the desired source. A widely-used technique for realizing suitable directional receiving characteristics has been to use the parabolic reflector, a rigid structure, generally a paraboloid of revolution, which concentrates the incoming sound power at its focal point where an electroacoustical transducer (microphone element) is located. For portable use in the field, the reflector must be of a convenient size to be carried or deployed, generally not much more than a meter in diameter. At this size, for sounds of frequencies that are efficiently propagated in the terrestrial atmosphere and are discernible to the human ear, the reflector is between approximately 0.5 and 30 wavelengths in diameter. In this range of dimensions the classical high-frequency (geometrical optics) and low-frequency (small-particle scattering) approximate methods of computation of the diffraction characteristics of material objects do not apply. However, with the availability of high speed computers with extremely large memory capacity, computational methods based on the classical Helmholtz integral equation formulation of the radiation problem have been developed using discrete-element approximations that can be made as precise as desired (Harrington 1993; Seybert et al. 1986; Jin 1993). Thus it is now possible to solve the mid-frequency diffraction problem with great accuracy, and to investigate small reflectors in considerable detail.

\section{Objective}

The objective of this study was to investigate the feasibility of using reflector microphones for field use as an effective and inexpensive alternative to linearray microphones. 


\section{Approach}

1. For this study, it was assumed that a hand-portable directional microphone system is desired for recording or observing outdoor sounds, for example, bird song, in the frequency range from, say, 200 to 5000 Hertz.

2. Three general techniques are examined: (a) the parabolic reflector, (b) the endfire transducer array or "shotgun" microphone, and (3) a novel system involving a plane reflector, the "baffle microphone."

3. Consideration was given to the way in which the performance of each of these systems varies with frequency, size of structure and, in the case of the parabola, with focal length.

4. Finally, attributes common to all three systems are discussed and conclusions are drawn regarding the feasibility of reflector microphones as alternatives to line-array microphones for field use.

\section{Mode of Technology Transfer}

It is anticipated that the results of this research will be used to design equipment configurations for studies that require use of outdoor microphones or microphone arrays, e.g., in noise abatement research. 


\section{Microphone Systems}

The directional attributes of a microphone system depend on the geometrical shape of its structure, its dimensions measured in wavelengths, and the disposition of its transducer or transducers. The wavelength depends on the frequency of the sound, in the relation:

$$
\mathbf{c}=v \lambda
$$

where:

$c=$ the speed of sound in air, here assumed to be $344 \mathrm{~m} /$ second

$v=$ the frequency in Hertz

$\lambda=$ the wavelength in meters.

A transducer is a device that converts the sound pressure or velocity in air to a voltage or current to drive an amplifier, recorder, or other output device. (The technical aspects of transducers are not addressed here.)

The directional characteristics are displayed as diagrams in polar coordinates, in which the relative sensitivity of the microphone to the sound amplitude is plotted as the radius, as a function of the angle with respect to the axis of the system. Here, the radius of the plot may be either linear in amplitude (sound pressure) or logarithmic in sound intensity. In the latter case, the unit used is the decibel $(\mathrm{dB})$. In the computations, it is assumed that the sound emanates from a point source at a great distance from the microphone. Each diagram applies only to a single frequency. This diagram gives a graphic conception of the reception "beam" of the microphone. Note that, in addition to the central lobe or "main beam" of the directional pattern, there are usually a number of "minor lobes" of lower strength. It is possible that, in recording sound from a source of unknown position, the sound may be received in one of these minor lobes, giving an entirely erroneous perception of both the direction and the strength of the source. 


\section{The Parabolic Reflector}

Parabolic reflectors are widely used in optical and radio engineering to provide directional characteristics in such diverse applications as automobile headlights, radar antennas, astronomical telescopes, and microwave communications. In all these cases, these reflectors are very large in terms of wavelengths to provide a high degree of directivity. Under this condition, the directional characteristics of the device can be calculated with reasonable accuracy by the classical methods of physical optics (Born and Wolf 1980), assuming specular reflection at the surface and certain approximations relating to the large size of the reflector.

In acoustics in the atmosphere, it is unusual to use a parabolic reflector that is large in terms of wavelengths, and that therefore possesses a narrow reception beam. Winds and spatial variations of temperature produce refraction, making the direction of arrival of the sound waves unpredictable and variable with time. Especially for portable use, it is preferable to use a small reflector with a relatively broad reception beam. A typical reflector sold for field use might be 0.5 to $1 \mathrm{~m}$ in diameter. In this size range, for the frequencies under discussion, the computation methods used extensively in radio and optical technology do not apply. Wahlstrom (1985) provides a practical discussion of this phenomenon that is useful for many purposes. However, to examine the details of the diffraction pattern of a small reflector, one must use a more precise computational technique.

Theoretically, the Method of Moments can be applied, with any degree of precision, to diffraction and scattering by arbitrarily-shaped structures. For this study, it was economical and convenient to use axial symmetry to reduce the computational burden. A program has been written (Lee 1996) for this purpose. A curve of the form:

$$
y+2 a=\sqrt{x^{2}+y^{2}}
$$

is assumed, in which the detector (transducer) is at the focal point at the origin and the surface is generated by rotation of the curve about the y-axis. The distance $a$ is the focal length. For each case, the source of sound is assumed to be 1000 wavelengths from the origin and the sound pressure is computed at the focal point. It is convenient to dimension the problem in terms of wavelength; then the results for a given case can be scaled to any frequency. For example, a source distance of 1000 wavelengths the reception pattern is essentially independent of the distance; this is the so-called "far field" situation. 
Figure 1 shows reception beams for a parabolic microphone one wavelength in diameter, which would be $50 \mathrm{~cm}$ at a frequency of $688 \mathrm{~Hz}$. Results are given for three different focal lengths, showing the critical nature of this parameter. Results are normalized so that the on-axis gain for each case is unity. The paraboloid with focal length 0.2 wavelength clearly has the most desirable pattern, with the smallest rearward lobe. The "front-to-back-ratio" for this case is 3.9 , or $11.8 \mathrm{~dB}$. (The power ratio is proportional to the square of the amplitude ratio.) For a shallow paraboloid (focal length 0.5 wavelength) the ratio is only 1.6 , or $4.2 \mathrm{~dB}$. The deepest paraboloid shown, focal length 0.15 wavelength, shows a distorted pattern with a lobe at about 120 degrees, $8.8 \mathrm{~dB}$ lower than the main lobe. The optimum focal length depends on the diameter in wavelengths, so a compromise is necessary for a system that is to accommodate a range of frequencies. For most applications, the highest front-to-back ratio is preferable to discriminate against sounds from unwanted directions. Note that all the patterns have minor lobes in addition to the main lobe, indicating some sensitivity to directions other than the desired one.

Figure 2 shows the performance of a paraboloid $47.6 \mathrm{~cm}$ in diameter with a focal length $30.3 \mathrm{~cm}$, for frequencies of 1,2 , and 3 kilohertz. Higher frequencies produce narrower beams. A rough rule of thumb applying to reflectors two or more wavelengths in diameter is that the amplitude beamwidth is approximately 60 degrees divided by the diameter in wavelengths. Here "beamwidth" is defined as the angle between the directions at which the gain is 0.707 times that on axis. Minor lobe structure is highly frequency dependent.

Figure 3 shows how the gain of a parabolic microphone depends on the frequency, for three different directions within the main beam. Note that a sound signal containing several different frequency components will not be faithfully represented in the output of the microphone, even if the reflector is accurately aimed at the source. For the situation in which the microphone has been mis-aimed, the lack of fidelity is more pronounced. As the frequency increases, the beam narrowing accentuates the loss of gain and the increasingly complex minor lobe structure causes strong fluctuations in gain. Although Figure 3 is specific to its particular reflector parameters, such considerations apply in general to all reflector microphones. Clearly, one cannot rely on directional microphones to yield accurate spectra of sound sources except possibly under rigidly controlled laboratory conditions. (See also Pye 1993). 


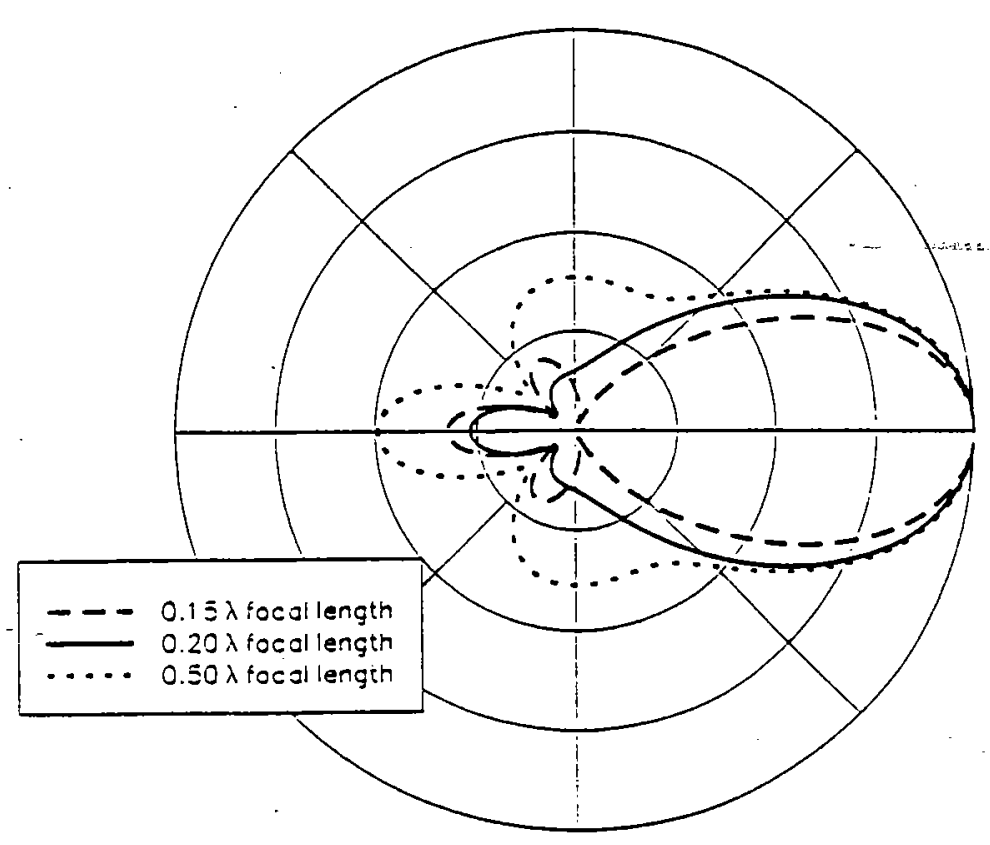

Figure 1. Reception patterns of paraboloidal microphone, one wavelength in diameter, showing effect of focal length. In each case, the transducer is at focal point.

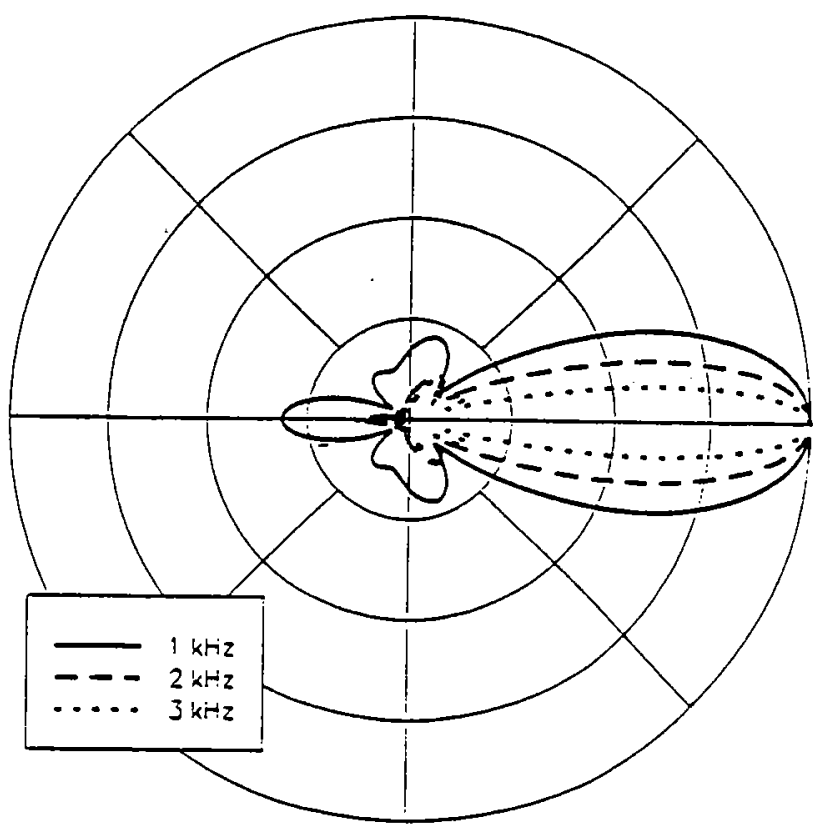

Figure 2. Reception patterns of a paraboloidal microphone, $47.6 \mathrm{~cm}$ in diameter, showing the effect of frequency on the pattern. Focal length is $30.3 \mathrm{~cm}$; transducer at focal point. 


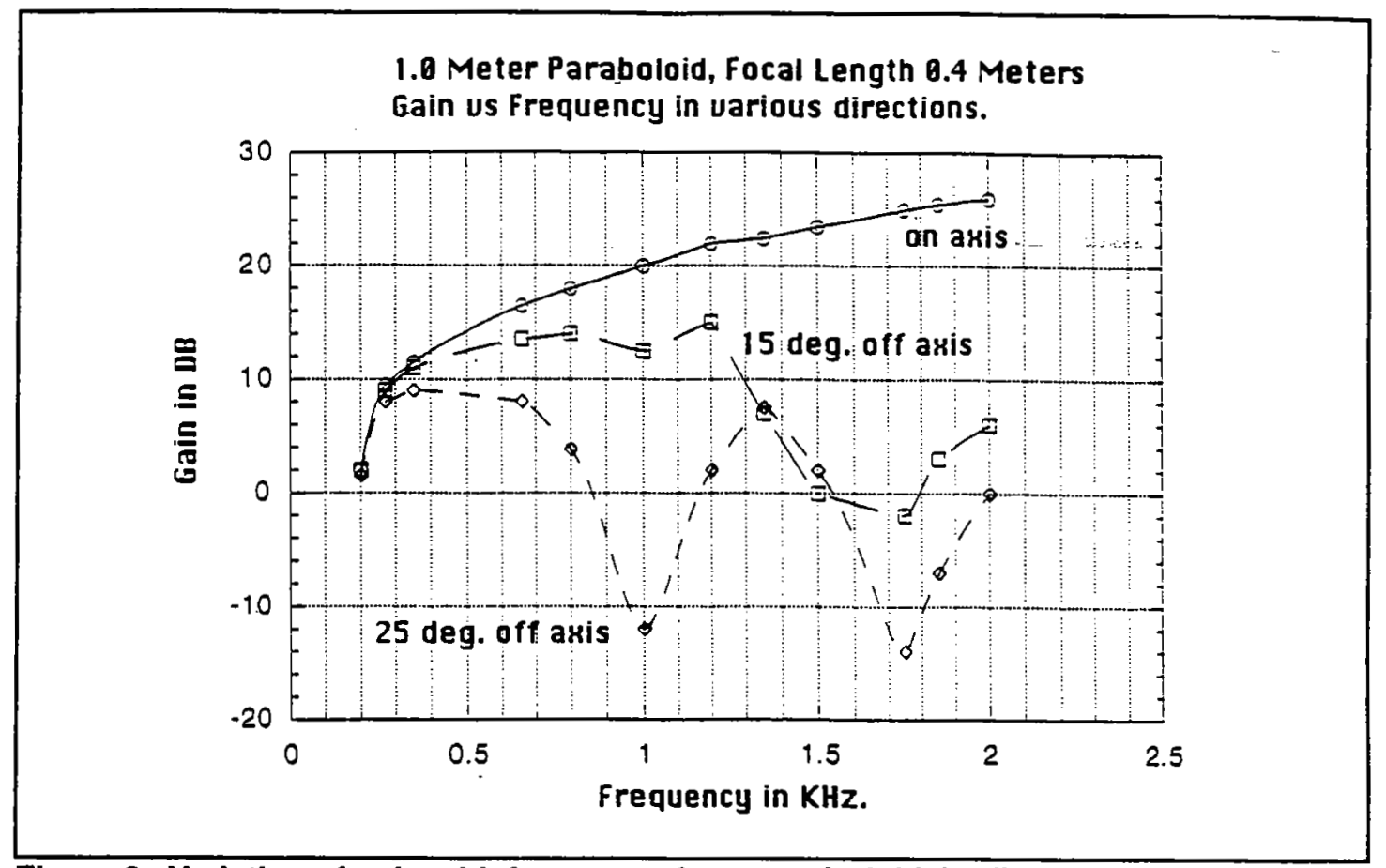

Figure 3. Variation of gain with frequency for a paraboloidal reflector 1.0 meter in dlameter, focal length $\mathbf{4 0} \mathrm{cm}$, at various directions within the main beam: on the principal axis, 15 degrees off axis, and 25 degrees off axis.

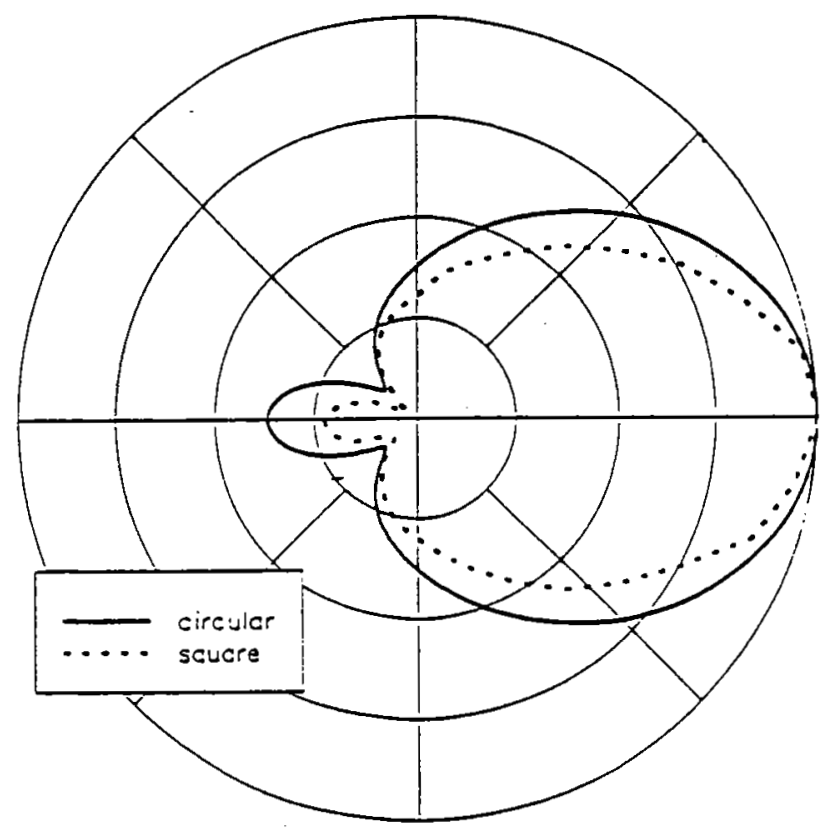

Figure 4. Reception patterns of flat reflector microphones, square and circular in shape and 0.89 wavelength in dimension. "Circular" pattern was computed; "Square" pattern was measured (after Benson et al., 1994). 


\section{The Flat Reflector Microphone}

Structures small in terms of wavelength do not scatter waves in narrow beams. By a well established reciprocity principle (Strutt 1877), it follows that a small microphone structure cannot have a highly directional reception pattern. From this it can be argued that, for a sufficiently small reflector, the specific shape of the reflector has only a small influence on the reception pattern. Thus, a small parabolic reflector may not be the most economical or convenient choice for field use, depending on the circumstances. Use of a flat reflector has been investigated as an easily-constructed means of achieving moderate directivity (Benson et al. 1994). The logical location for the transducer in such an arrangement would be directly on the reflector surface, as the sound pressure at that point is double the pressure in the incident wave. Figure 4 shows the computed sound pressure reception pattern of a flat circular disk 0.889 wavelength in diameter with a transducer on the surface at the center. Also shown is the measured pattern of a square, flat panel of the same dimension. This measured pattern is almost identical to the pattern computed by the Method of Moments (Boag 1994; Lee 1996), confirming the accuracy of the computational method.

Figure 5 shows the effect of frequency on the reception pattern of the circular disk, here assumed to be $16 \mathrm{~cm}$ in diameter. If the reflectors of Figure 4 are also assumed to be $16 \mathrm{~cm}$ in dimension, their patterns are those obtaining at 1911 $\mathrm{Hz}$. This simple device gives useful directivity and reasonable pattern shapes, though at the lowest frequency, the scalloping of the principle lobe amounts to $7.0 \mathrm{~dB}$. For the experimental measurements the reflectors were sawed from 1.3$\mathrm{cm}$ thick particleboard and the transducers were very inexpensive electret microphone elements fastened directly to the surface.

Figure 6 shows a flat-reflector microphone designed for the frequency band 10 to $40 \mathrm{~Hz}$. Such low frequencies are characteristic of the sounds produced by explosions, and the device illustrated was constructed to monitor the environmental noise of a military artillery training facility. The dimensions of the reflector are $4.5 \times 9.0 \mathrm{~m}$, and the microphone is mounted at ground level immediately in front of the surface. The system, including the ground surface, which is assumed impermeable at these frequencies, is equivalent (with respect to its reception pattern) to a $9.0 \mathrm{~m}$ square reflector in unbounded air. 


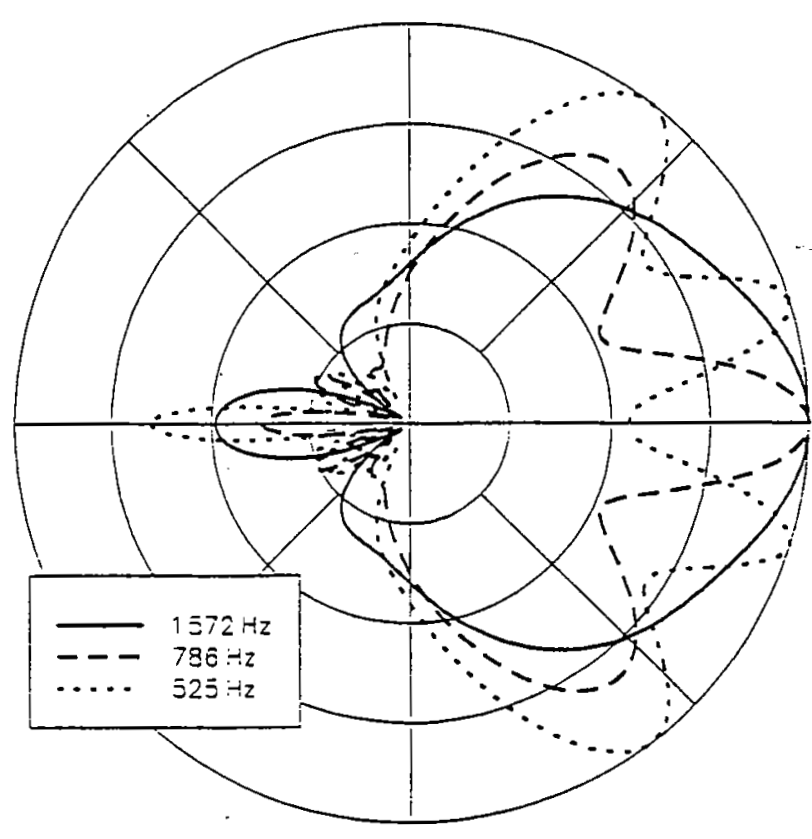

Figure 5. Reception patterns of a flat disk microphone, $16 \mathrm{~cm}$ in diameter, showing effect of frequency. Transducer is on the surface at the center of the disk.

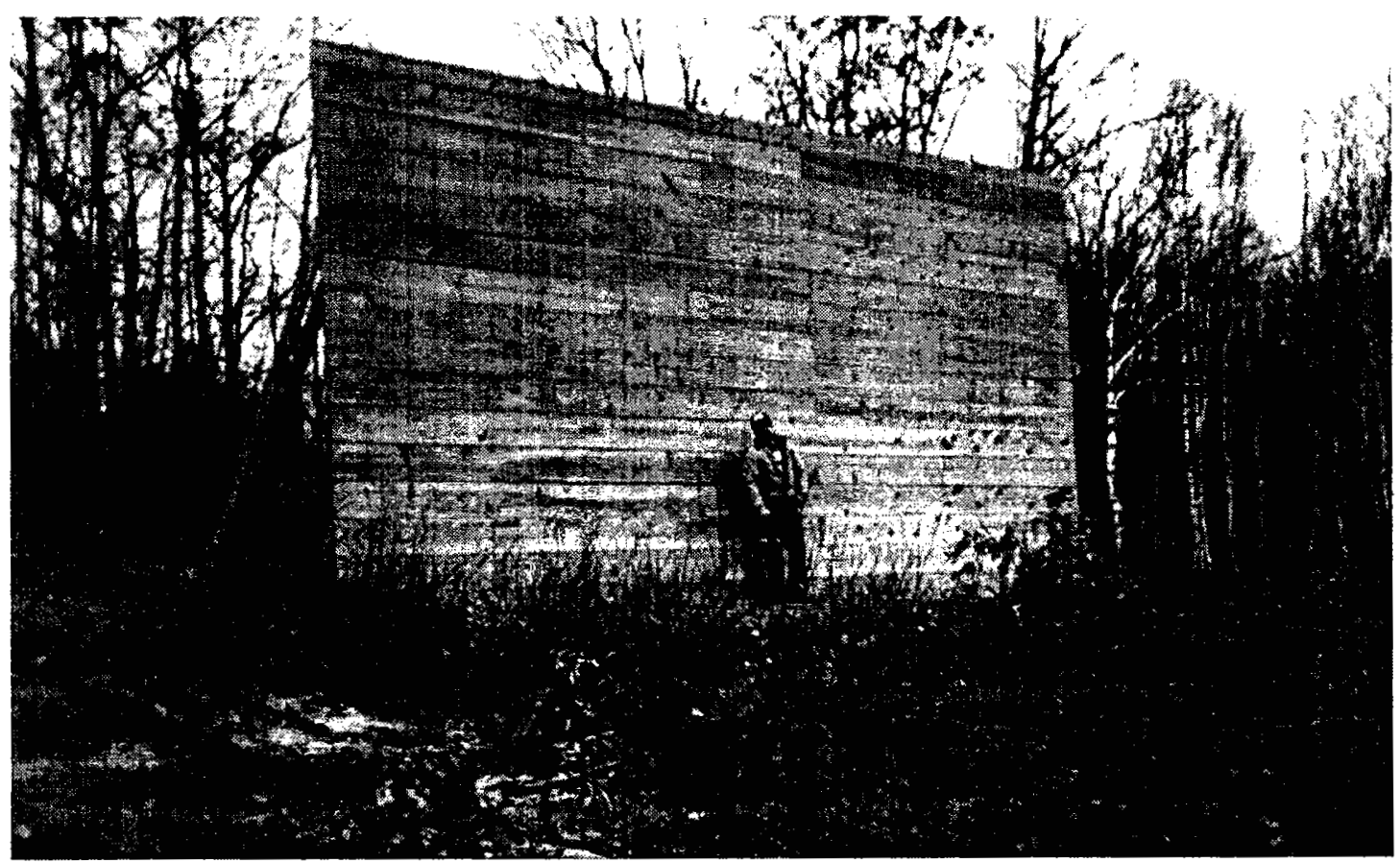

Figure 6. A flat reflector microphone baffle for low frequencies (10 to $40 \mathrm{~Hz}$ ). 


\section{End-Fire Microphones}

The end-fire microphone or "shotgun" microphone, a popular device for portable use, is briefly discussed here for comparison with reflector microphones. In its canonical form, it consists of an array of two or more transducers along an axis, some or all of which are associated with phase-shift or time-delay arrangements to optimize the response in the axial direction (Olson 1957; 1972). Any end-fire array has an inherently broader reception pattern than a reflector microphone of the same linear dimension. The reception pattern of an array is easily analyzed. Figure 7 (solid curve) shows the pattern of an array consisting of two transducers separated by one-fourth wavelength with an appropriate acoustical time delay in the input to one of them to optimize the response in the axial direction. Here, the outputs of the transducers are assumed to be weighted equally in amplitude.

At a frequency of $500 \mathrm{~Hz}$, the length of the device would be $17.25 \mathrm{~cm}$. This microphone would be useful in many situations, since it is compact, easily portable, and would not require precise aiming. At twice this frequency, the same device would have a different pattern, as shown in the dashed curve in Figure 7.

An array of four transducers produces the reception patterns shown in Figure 8. For the dotted curve, the separation between transducers is 0.2 wavelength; for the dashed curve, 0.3 wavelength; and for the solid curve, 0.75 wavelength. Thus, it is apparent that, in the linear-array or "shotgun" microphone, it is important that the spacing between elements be less than 0.5 wavelength, at the highest frequency to be observed, if a single-lobed pattern is to be achieved.

In practice, the shotgun microphone may be constructed of a number of tubes feeding a common transducer, or of a single tube perforated at intervals to admit pressure components at successive points along the tube, which in turn feeds a transducer at its end. These arrangements are artfully designed to produce useful patterns across a broad range of frequencies, and even to optimize the irregular amplitude response with frequency that is basically inherent with any (mis-aimed) directional microphone. However, the engineering effort required to achieve these attributes results in high cost for the manufactured item: 


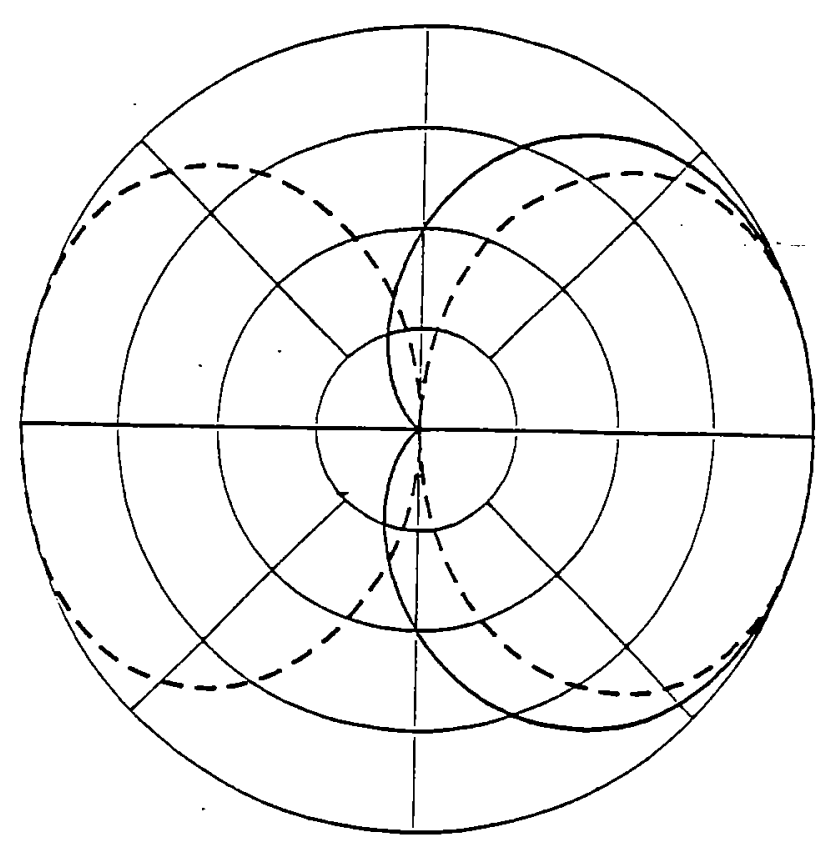

Figure 7. Reception patterns of an array of two transducers. Dashed curve: separation one-fourth wavelength; solid curve: one-half wavelength. In each case a time delay corresponding to the separation is inserted into the signal path of one transducer.

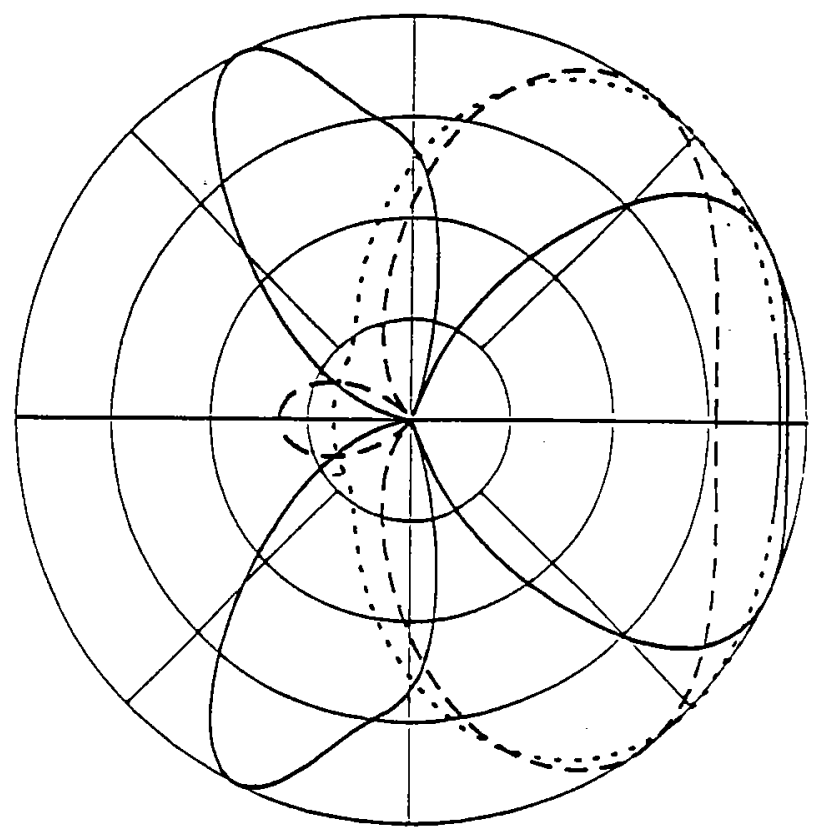

Figure 8. Reception patterns of a four-element array, at frequencies corresponding to inter-element spacings of 0.2 wavelength (dotted), 0.3 wavelength (dashed), and 0.75 wavelength (solid). 


\section{Conclusion}

Reflector microphones may provide inexpensive and effective alternatives to linearray microphones for field use; however it should be appreciated that all directional microphones have definite limitations with respect to fidelity of sound reproduction. The line-array (shotgun) microphone can be engineered to reduce its frequency dependent gain and pattern variations, but at substantial increase in cost.

The physical environment is also a factor in microphone performance. Wind noise is troublesome although it can be mitigated somewhat by use of windscreens. Nearby obstacles such as the ground, buildings, persons, etc., especially in ad hoc situations, also produce essentially unpredictable variations in gain and pattern. This study concludes that it is not productive to seek the ultimate in performance from a microphone system for use in the field, and that these limitations must be considered in interpreting field data. 


\section{References}

Benson, J.W., Y.L. Li, and G.W. Swenson, Jr., “A Baffle-Type Directional Microphone,” J. Acoust. Soc. Am., vol 5 (1994), pp 2536-2538.

Boag, Alona, Unpublished notes (1994).

Born, M., and E. Wolf, Principles Of Optics, $6^{\text {th }}$ ed. (Pergamon Press, Oxford, 1980).

Harrington, R.F., Field Computation by Moment Methods (IEEE Press, Piscataway, NJ, 1993).

Lee, Soon-Young, Diffraction of Sound by Objects in the One-Wavelength Size Range, Master's Thesis (Dept. of Electrical Engineering, University of Illinois at Urbana-Champaign, 1996).

Olson, H.F., Acoustical Engineering, $2^{\text {nd }}$ ed. (D. Van Nostrand Co., Princeton, NJ, 1957).

Olson, H.F., Modern Sound Reproduction (Van Nostrand Reinhold, New York 1972).

Pye, J.D., "Is Fidelity Futile? The "True" Signal Is Illusory, Especially With Ultrasound," Bioacoustics, vol 4, pp 271-286 (1993).

Strutt, J.W. (Lord Rayleigh) The Theory of Sound (Macmillan, London, 1877; Dover Edition, New York, 1945).

Swenson, G.W., Jr., Principles of Modern Acoustics (D. Van Nostrand Co., New York, 1956).

Wahlström, S., "The Parabolic Reflector as an Acoustical Amplifier," J. Audio Eng. Soc., vol 33 (1985), pp 419-429. 